\title{
Robust Design of Dead-Time Compensator Controllers for Constrained Non-Linear Systems
}

\author{
Daniel Limón, Martín Pomar, Julio E. Normey-Rico, Tito L. M. Santos and E.F. Camacho
}

\begin{abstract}
This paper presents a predictor based approach to design robust controllers for nonlinear dead-time systems. A dead-time compensation structure is proposed for constrained control systems with bounded disturbances and dead-time. It is shown that input-to-state stability (ISS) and constraint satisfaction of the controlled system can be guaranteed if the control law stabilizes (in the ISS sense) an equivalent nonlinear dead-time free system. This result allows the simplification of the controller synthesis which is performed for the dead-time free model of the process. Some simulation results are used to illustrate the performance obtained with the proposed scheme.
\end{abstract}

\section{INTRODUCTION}

Time delays, also known as dead-times, can be found in most industrial processes dynamics. Dead-times are caused mainly by the time required to transport mass, energy or information, but they can also be caused by processing time or by the accumulation of time lags in a number of simple dynamic systems connected in series [1]. Processes with delay are difficult to control using traditional controllers mainly because of the following [2]: (i) the effect of the disturbances is not noticed until a considerable time has elapsed, (ii) the effect of the control action takes some time to be noticed in the controlled variable, and (iii) the control action that is applied based on the actual error tries to correct a situation that originated some time before.

The undesired effects caused by dead-time in the closedloop system may be avoided using predictor based controllers, which are extensions of the Smith Predictor [3]. The main idea of these schemes is to remove dead-time from the control loop by using a prediction of the process output [2].

Robust control of linear time delay systems has been widely studied for the unconstrained case, see [1], [4] and references therein. The case of input constrained systems with disturbances is analyzed in [5], [6], [7], [8], however, considering specific control strategies, and not a general dead-time compensation scheme. In practice, dead-time com-

Daniel Limón and E.F. Camacho are with Departamento de Ingeniería de Sistemas y Automática, Universidad de Sevilla, Avda. Camino de los Descubrimientos s/n, 41092 Sevilla, España. $\{$ Iimon, eduardo\}@cartuja.us.es

Martín Pomar, Tito L. M. Santos and Julio E. Normey-Rico is with Departamento de Automação e Sistemas, Universidade Federal de Santa Catarina, Caixa Postal 476, CEP 88040-900, Florianópolis, Santa Catarina, Brasil.mpogar, tito, julio@das.ufsc.br

First and fifth authors acknowledge the European 7th framework STREP project "Hierarchical and distributed model predictive control (HD-MPC)" (contract number INFSO-ICT-223854) as well as the Network of Excellence HYCON2 (grant agreement $n^{\circ} 257462$ ). Second and third author were funded by Projeto CAPES/DGU $\mathrm{N}^{\circ}$ 220/2010. And fourth author were founded by Conselho Nacional de Desenvolvimento Científico e Tecnológico (CNPq) pensation can be performed explicitly, outside the optimization problem, in order to simplify the control algorithm or to improve robustness [1]. This kind of prediction is based on a nominal model, and disturbance effects are considered to guarantee robust stability and constraint satisfaction. These ideas are used in [9] to propose a robust MPC for constrained dead-time systems.

Conceptually dead-time compensation structures used in the linear case can be applied to nonlinear dead-time processes, that is, the controller used in the dead-time free system can be used in the delayed system through a proper dead-time compensator. This idea was used to obtain adequate practical results in several works, however without stability proofs [1]. Thus, this work presents a dead-time compensator robust control approach that can be used with nonlinear systems with bounded additive uncertainties. The proposed controller allows to extend the results obtained for the linear case in [9] and mainly uses two principal ideas: an explicit nonlinear dead-time compensation and a procedure to bound the prediction error [10].

The paper is organized as follows. Problem statement and dead-time compensation structure are presented, respectively, in sections II and III. Section IV is dedicated to the stability analysis where the main result is presented. Simulation examples are presented in Section $\mathrm{V}$ and the concluding remarks are discussed in Section VI.

\section{Notation}

For a certain signal $v, v_{k}$ denotes the value of the signal at sampling instant $k$ and $\mathbf{v}=\left\{v_{0}, v_{1}, v_{2}, \cdots\right\}$ denotes a time sequence of the signal $v$. For given positive integers $i$ and $j$ such that $i<j, \mathbf{v}_{[i, j]}=\left\{v_{i}, \cdots, v_{j}\right\} . \mathbf{0}$ denotes the sequence of a null signal with an appropriate dimension. For a given sequence $\mathbf{v},\|\mathbf{v}\|_{s}=\sup _{k}\left\{\left\|v_{k}\right\|_{s}\right\}$, where $\|\cdot\|_{s}$ denotes certain norm. Given a set $Z \in \mathbb{R}^{p}$ and a constant $\gamma \geq 0, \gamma Z=\{\gamma z: z \in Z\}$. For a given couple of sets $A, B \subseteq \mathbb{R}^{n}$, the Pontryagin difference $A \ominus B$ is defined as the set $\{x: x+b \in A, \forall b \in B\}$.

$\mathbb{R}_{+}$denotes the set of reals that are positive or equal to zero. A function $\alpha: \mathbb{R}_{+} \rightarrow \mathbb{R}_{+}$is a $\mathcal{K}$ function if it is continuous in its domain, $\alpha(0)=0$ and it is strictly increasing. A function $\alpha: \mathbb{R}_{+} \rightarrow \mathbb{R}_{+}$is a $\mathcal{K}_{\infty}$ function if it is a $\mathcal{K}$ function and $\lim _{s \rightarrow \infty} \alpha(s)=\infty$. A function $\beta: \mathbb{R}_{+} \times \mathbb{R}_{+} \rightarrow \mathbb{R}_{+}$is a $\mathcal{K} \mathcal{L}$ function if $\beta(t, s)$ is continuous, is a $\mathcal{K}$ function in $s$ for all $t \geq 0$, strictly decreasing in $t$ for all $s \geq 0$ and $\lim _{t \rightarrow \infty} \beta(t, s)=0$ for all $s \geq 0$. 


\section{SYSTEM DESCRIPTION}

Consider an uncertain nonlinear discrete time system with dead-time given by

$$
x_{k+1}=f\left(x_{k}, u_{k-d}\right)+w_{k}
$$

where $x_{k} \in \mathbb{R}^{n}$ is the state of the system, $u_{k} \in \mathbb{R}^{m}$ is the control vector, $w_{k}$ the additive uncertainty and $d$ is the dead-time.

The system is subject to hard constraints on the state and on the control action

$$
\begin{aligned}
& x_{k} \in X, \\
& u_{k} \in U
\end{aligned}
$$

where $X$ and $U$ are closed sets, both of them containing the origin.

The additive uncertainty vector $w_{k} \in \mathbb{R}^{n}$ is known to be bounded in a compact set $W$ that contains the origin,

$$
w_{k} \in W
$$

Notice that the additive uncertainty can model perturbed systems and a wide class of model mismatch if the following condition holds:

$$
w_{k}=\left[x_{k+1}-f\left(x_{k}, u_{k-d}\right)\right] \in W, \forall x_{k} \in X, u_{k} \in U
$$

The only assumption on $\mathrm{W}$ is that it is a compact set [10].

The aim of this paper is to derive controllers to be designed ignoring the delay but in such a way that constrained robust stability holds in presence of dead-time. Then, it is useful to define the model of the plant without the dead-time effect

$$
\tilde{x}_{k+1}=f\left(\tilde{x}_{k}, u_{k}\right)+w_{k}
$$

The solution of the system (5) for a initial state $x$, a sequence of inputs $\mathbf{u}$ and disturbances $\mathbf{w}$ at sampling time $k$ is denoted as

$$
\tilde{\phi}(k, x, \mathbf{u}, \mathbf{w})
$$

Then, taking into account the dynamics of the dead-time we have that

$$
x_{k}=\tilde{\phi}\left(k-d, x_{d}, \mathbf{u}_{[0, k-d-1]}, \mathbf{w}_{[d, k-1]}\right)
$$

Note that $x_{d}$ acts as initial state since it is the most recent state that does not depend on the sequence $\mathbf{u}$.

Ignoring the effect of the uncertainty in the plant, the nominal trajectory is obtained. This is denoted, with an slight abuse of notation, as

$$
\tilde{\phi}\left(k, x, u_{[0, k-1]}\right)=\tilde{\phi}\left(k, x, \mathbf{u}_{[0, k-1]}, \mathbf{0}\right)
$$

\section{EXPLICIT DEAD-TIME COMPENSATION}

A simple idea discussed in [11] can be applied to consider a different representation of the system without dead-time. From Eq. 1, we can see that there is no effect of $u_{k}$ over $x_{k+1}, x_{k+2}, \ldots, x_{k+d}$ due to the dead-time. As consequence, in absence of uncertainties, namely $\mathbf{w}=\mathbf{0}, x_{k+d}$ depends only on past controls, so this can be obtained from the function $\tilde{\phi}$ knowing the current state of the plant $x_{k}$ and the input sequence $\mathbf{u}_{[k-d, k-1]}$.

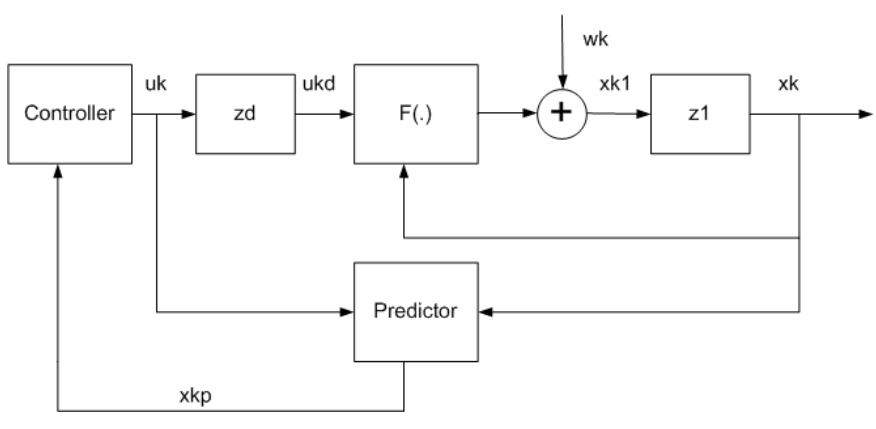

Fig. 1. Controller with explicit delay compensation structure.

Denoting the predicted state by $\hat{x}_{k}$, we have that

$$
\hat{x}_{k}=\tilde{\phi}\left(d ; x_{k}, \mathbf{u}_{[k-d, k-1]}\right)
$$

So, through a simple change of variable we can represent the system (without uncertainty) cascaded by the deadtime compensator by the following state-space model of the system without dead-time

$$
\hat{x}_{k+1}=f\left(\hat{x}_{k}, u_{k}\right)
$$

As it is well known since Smith's seminal work [3], in the absence of uncertainty, i.e. $\mathbf{w}=\mathbf{0}$, the real state at sample time $k+d, x_{k+d}$, is equal to the predicted state $\hat{x}_{k}$. Then, in absence of uncertainty, a control law $u_{k}=\kappa\left(\hat{x}_{k}\right)$ such that stabilizes the dynamical model (9), also stabilizes the system (1).

However, when $\mathbf{w} \neq \mathbf{0}$, the former control structure, depicted in figure 1 , does not ensure that $x_{k+d}=\hat{x}_{k}$, since in general $x_{k+d} \neq \tilde{\phi}\left(d ; x_{k}, u_{[k-d, k-1]}\right)$. Then the stabilizing design of the control law $u_{k}=\kappa\left(\hat{x}_{k}\right)$ may not guarantee the robust stability of the closed-loop system.

The objective of this paper is to analyze the effect of uncertainties on the dead-time compensator for an uncertain non-linear system. Based on this, conditions to design the control law to ensure robust stability and robust constraint satisfaction of the whole closed-loop uncertain system will be derived.

\section{A. Analysis the effect of the uncertainty on the dead-time compensator}

Since the values that the uncertainty can take are unknown, it is impossible to predict accurately the state of the real system. In this section we show that, based on the set of possible values that the uncertainty can take $(W)$, the effect of the uncertainty on the predicted state can be bounded. Furthermore, it is demonstrated that the uncertain system cascaded with the dead-time compensator can be posed as a dead-time free system with additive bounded uncertainties as follows

$$
\hat{x}_{k+1}=f\left(\hat{x}_{k}, u_{k}\right)+\hat{w}_{k}
$$

where $\hat{x}_{k}$ is given by (8).

We consider the following assumption on the model of the system 
Assumption. 1: The model function $f(x, u)$ is such that $f(0,0)=0$ and it is locally Lipschitz in $x$ in the domain $X \times U$, i.e. there is a constant $0<L_{f}<\infty$ such that for all $x_{1}, x_{2} \in X$ and for all $u \in U$,

$$
\left\|f\left(x_{1}, u\right)-f\left(x_{2}, u\right)\right\|_{s} \leq L_{f} \cdot\left\|x_{1}-x_{2}\right\|_{s}
$$

The constant $L_{f}$ denotes the Lipschitz constant in a given norm denoted s-norm.

It is worth remarking that the Lipschitz continuity of a function does not depend on the chosen norm in (11), but the value of the Lipschitz constant does.

Under this assumption, the following lemma can be derived

Lemma. 1: Consider that assumption 1 holds. Then the function $\tilde{\phi}(j, x, \mathbf{u})$ is Lipschitz continuous in $x$, for all $j \geq 0$, $x \in X$ and $u_{i} \in U$. Furthermore $L_{f}^{j}$ is a Lipschitz constant value.

Proof: This lemma is proved by demonstrating that the following property holds

$$
\left\|\tilde{\phi}\left(j, a, \mathbf{u}_{[0, j-1]}\right)-\tilde{\phi}\left(j, b, \mathbf{u}_{[0, j-1]}\right)\right\|_{s} \leq L_{f}^{j}\|a-b\|_{s}
$$

This will be proved by recursion. For $j=1$ this is immediate observing that $\tilde{\phi}\left(1, a, u_{0}\right)=f\left(a, u_{0}\right)$. Assume that the property holds for $j-1$, then taking into account that

$$
\tilde{\phi}\left(j, a, \mathbf{u}_{[0, j-1]}\right)=f\left(\tilde{\phi}\left(j-1, a, \mathbf{u}_{[0, j-2]}\right), u_{j-1}\right)
$$

we have that

$$
\begin{aligned}
\left\|\tilde{\phi}\left(j, a, \mathbf{u}_{[0, j-1]}\right)-\tilde{\phi}\left(j, b, \mathbf{u}_{[0, j-1]}\right)\right\|_{s} & \leq \\
L_{f}\left\|\tilde{\phi}\left(j-1, a, \mathbf{u}_{[0, j-2]}\right)-\tilde{\phi}\left(j-1, b, \mathbf{u}_{[0, j-2]}\right)\right\|_{s} & \leq \\
L_{f}^{j}\|a-b\|_{s} &
\end{aligned}
$$

Based on this lemma, the following theorem is stated.

Theorem. 1: Let a system be described by (1) and let the predicted state $\hat{x}_{k}$ given by (8). The dynamics of the predicted state is given by

$$
\hat{x}_{k+1}=f\left(\hat{x}_{k}, u_{k}\right)+\hat{w}_{k}
$$

with $\hat{w}_{k} \in L_{f}^{d} W$ and $\hat{x}_{0}$ given by (8) for $k=0$.

Proof: By (8) we have that

$$
\hat{x}_{k+1}=\tilde{\phi}\left(d ; x_{k+1}, u_{[k+1-d, k]}\right)
$$

and additionally

$$
\begin{aligned}
f\left(\hat{x}_{k}, u_{k}\right) & =f\left(\tilde{\phi}\left(d ; x_{k}, u_{[k-d, k-1]}\right), u_{k}\right) \\
& =\tilde{\phi}\left(d+1 ; x_{k}, u_{[k-d, k]}\right) \\
& =\tilde{\phi}\left(d ; f\left(x_{k}, u_{k-d}\right), u_{[k+1-d, k]}\right)
\end{aligned}
$$

Then, since $\hat{w}_{k}=\hat{x}_{k+1}-f\left(\hat{x}_{k}, u_{k}\right)$, in virtue of lemma 1 , we infer that

$$
\begin{aligned}
\left\|\hat{w}_{k}\right\|_{s} & =\left\|\hat{x}_{k+1}-f\left(\hat{x}_{k}, u_{k}\right)\right\|_{s} \\
& \leq L_{f}^{d}\left\|x_{k+1}-f\left(x_{k}, u_{k-d}\right)\right\|_{s} \\
& =L_{f}^{d}\left\|w_{k}\right\|_{s}
\end{aligned}
$$

Therefore, it can be derived that

$$
\left\|\hat{w}_{k}\right\|_{s} \leq L_{f}^{d} \gamma
$$

Since $W=\{w:\|w\| \leq \gamma\}$, we infer that $\left\|\hat{w}_{k}\right\|_{s} \leq L_{f}^{d} \gamma$ and then $\hat{w}_{k} \in L_{f}^{d} W$.

This result is important because this allows to design controllers based on $\hat{x}_{k}$. In order to derive the stability property and constraint satisfaction, it would be interesting to calculate a bound of the real state $x_{k}$ and the estimated $\hat{x}_{k}$. Some important consequences of this fact will be further discussed.

\section{B. Bounding dead-time compensator error}

Since there are mismatches between the real system and the nominal model, the computed states using a nominal model might differ from the real states of the system. In order to consider this effect in the controller synthesis, a bound on this difference will be computed. This bound is based on the following lemma, based on the results of [10].

Lemma. 2: Consider that system (1) fulfils assumption 1 and let $\tilde{\phi}$ be the function given in (6). Consider a given initial state $z_{0}$ and a couple of sequences $\mathbf{u}$ and $\mathbf{w}$ and define

$$
\begin{aligned}
& z_{k}=\tilde{\phi}\left(k, z 0, \mathbf{u}_{[0, k-1]}, \mathbf{w}_{[0, k-1]}\right) \\
& \bar{z}_{k}=\tilde{\phi}\left(k, z 0, \mathbf{u}_{[0, k-1]}\right)
\end{aligned}
$$

then we have that

$$
\left\|z_{k}-\bar{z}_{k}\right\|_{s} \leq \frac{L_{f}^{k}-1}{L_{f}-1}\left\|\mathbf{w}_{[0, k-1]}\right\|_{s}
$$

Proof: This is proved by recursion. It is immediate to see that for $k=0$ the condition holds. Assume that it holds for $k-1$, that is,

$$
\left\|z_{k-1}-\bar{z}_{k-1}\right\|_{s} \leq \frac{L_{f}^{k-1}-1}{L_{f}-1}\left\|\mathbf{w}_{[0, k-2]}\right\|_{s}
$$

Then

$$
\begin{aligned}
\left\|z_{k}-\bar{z}_{k}\right\|_{s} & =\left\|f\left(z_{k-1}, u_{k-1}\right)+w_{k-1}-f\left(\bar{z}_{k-1}, u_{k-1}\right)\right\|_{s} \\
& \leq L_{f}\left\|z_{k-1}-\bar{z}_{k-1}\right\|_{s}+\left\|w_{k-1}\right\|_{s} \\
& \leq L_{f} \frac{L_{f}^{k-1}-1}{L_{f}-1}\left\|\mathbf{w}_{[0, k-2]}\right\|_{s}+\left\|w_{k-1}\right\|_{s} \\
& \leq\left(L_{f} \frac{L_{f}^{k-1}-1}{L_{f}-1}+1\right)\left\|\mathbf{w}_{[0, k-1]}\right\|_{s} \\
& =\frac{L_{f}^{k}-1}{L_{f}-1}\left\|\mathbf{w}_{[0, k-1]}\right\|_{s}
\end{aligned}
$$

Based on this lemma, the bound between the real state of the uncertain system and the predicted one is calculated. This is stated in the following theorem.

Theorem. 2: Consider a system (1) such that assumption 1 is satisfied. Then, for a given sequence of inputs $\mathbf{u}$ and 
uncertainty $\mathbf{w}$, the difference between the states of the deadtime compensator at sample time $k-d, \hat{x}_{k-d}$, and the real state of the system $x_{k}$ is bounded by

$$
\left\|x_{k}-\hat{x}_{k-d}\right\|_{s} \leq \frac{L_{f}^{d}-1}{L_{f}-1}\left\|\mathbf{w}_{[k-d, k-1]}\right\|_{s}
$$

Proof: Notice that

$$
\begin{aligned}
x_{k} & =\tilde{\phi}\left(k-d, x_{d}, \mathbf{u}_{[0, k-d-1]}, \mathbf{w}_{[d, k-1]}\right) \\
& =\tilde{\phi}\left(d, x_{k-d}, \mathbf{u}_{[k-2 d, k-d-1]}, \mathbf{w}_{[k-d, k-1]}\right)
\end{aligned}
$$

and

$$
\hat{x}_{k-d}=\tilde{\phi}\left(d, x_{k-d}, \mathbf{u}_{[k-2 d, k-d-1]}\right)
$$

Then, in virtue of lemma 2 we have that

$$
\left\|x_{k}-\hat{x}_{k-d}\right\|_{s} \leq \frac{L_{f}^{d}-1}{L_{f}-1}\left\|\mathbf{w}_{[k-d, k-1]}\right\|_{s}
$$

and the theorem is proved.

Notice that the computed bound may be over-conservative due to the global nature of the Lipschitz constant used. This bound can be reduced considering some procedures as shown in [10].

Therefore, the dead-time compensator error,

$$
e_{k}=x_{k}-\hat{x}_{k-d}
$$

has a cumulative effect and belongs to the set

$$
E=\left\{e \in \mathbb{R}^{n}:\|e\|_{s} \leq \frac{L_{f}^{d}-1}{L_{f}-1} \cdot \gamma\right\}
$$

In the next section we will be developed results on closed loop robust stability of dead-time systems based on deadtime compensators. These results are based on the last two theorems, and they are the main contribution of this paper.

\section{STABILITY ANALYSIS}

\section{A. Input-to-state stability}

The concept of input-to-state stability (ISS) has been widely used in stability analysis and control synthesis of nonlinear system [12]. Some of the well established results in ISS for continuous time nonlinear system have been extended to discrete-time nonlinear systems in [13]. Some of the results used in controller synthesis based on deadtime compensator presented in this paper are shown in this section.

Definition. 1 (Jiang \& Wang [13]): Consider a system given by

$$
x_{k+1}=F\left(x_{k}, w_{k}\right)
$$

where $x_{k}$ is the state of the system, and $w_{k}$ a bounded input (disturbance) of the system such that $w_{k} \in W$ for all $k$. Then the system is ISS if there are a $\mathcal{K} \mathcal{L}$-function $\beta(\cdot, \cdot)$ and a $\mathcal{K}$-function $\delta(\cdot)$ such that

$$
\left\|x_{k}\right\| \leq \beta\left(x_{0}, k\right)+\delta\left(\left\|\mathbf{w}_{[0, k-1]}\right\|\right)
$$

Note that an ISS system is asymptotically stable in absence of input or if the input is decaying. Moreover, it has been proved that this is equivalent to the existence of a stability margin for the system [13]. If the input is merely bounded then the evolution of the system is ultimately bounded in a set which size depends on the bound of the input.

Input-to-state stability notion has demonstrated to be a suitable framework to the robust stability analysis of constrained uncertain systems [14], and this will be used in this paper for the robust stability results.

\section{B. Main result}

Now we are ready to state our main result: a input-tostate stabilizing control law designed ignoring the dead-time can be used to control de plant with dead-time by adding a dead-time compensator.

In effect, consider that a control law $\kappa: \mathbb{R}^{n} \rightarrow \mathbb{R}^{m}$ has been designed. Then the controlled system with the deadtime compensator is given by the following equations

$$
\begin{aligned}
x_{k+1} & =f\left(x_{k}, u_{k-d}\right)+w_{k} \\
\hat{x}_{k} & =\tilde{\phi}\left(d, x_{k}, \mathbf{u}_{[k-d, k-1]}\right) \\
u_{k} & =\kappa\left(\hat{x}_{k}\right)
\end{aligned}
$$

Then, in the following theorem sufficient conditions to design the control law $\kappa$ to ensure that the controlled system is input-to-state stable and that the constraints are robustly fulfilled.

Theorem. 3:

(i) If the control law $u_{k}=\kappa\left(\hat{x}_{k}\right)$ is such that the system

$$
\hat{x}_{k+1}=f\left(\hat{x}_{k}, \kappa\left(\hat{x}_{k}\right)\right)+\hat{w}_{k}
$$

is ISS for all $\hat{w}_{k} \in L_{f}^{d} W$, then the controlled system (17) is ISS for all $w_{k} \in W$.

(ii) Besides, if the set $X \ominus E$ is not empty and the control law $u_{k}=\kappa\left(\hat{x}_{k}\right)$ is such that $\hat{x}_{k} \in X \ominus E$ for all $k \geq 0$, then $x_{k} \in X$ for all $k \geq d$.

Proof:

Since the system $\hat{x}_{k+1}=f\left(\hat{x}_{k}, \kappa\left(\hat{x}_{k}\right)\right)+\hat{w}_{k}$, there exists a $\mathcal{K} \mathcal{L}$ function $\beta$ and a $\mathcal{K}$ function $\sigma$ such that

$$
\left\|\hat{x}_{k}\right\|_{s} \leq \beta\left(k,\left\|\hat{x}_{0}\right\|_{s}\right)+\sigma\left(\left\|\hat{\mathbf{w}}_{[0, k-1]}\right\|_{s}\right)
$$

From (12) it is derived that

$$
\left\|\hat{\mathbf{w}}_{[0, k-1]}\right\|_{s} \leq L_{f}^{d}\left\|\mathbf{w}_{[0, k-1]}\right\|_{s}
$$

and hence

$$
\left\|\hat{x}_{k}\right\|_{s} \leq \beta\left(k,\left\|\hat{x}_{0}\right\|_{s}\right)+\sigma\left(L_{f}^{d}\left\|\mathbf{w}_{[0, k-1]}\right\|_{s}\right)
$$

Taking into account that $e_{k}=x_{k}-\hat{x}_{k-d}$, we have that

$$
\begin{aligned}
\left\|x_{k}\right\|_{s}= & \left\|\hat{x}_{k-d}+e_{k}\right\|_{s} \\
\leq & \left\|\hat{x}_{k-d}\right\|_{s}+\left\|e_{k}\right\|_{s} \\
\leq & \beta\left(k-d,\left\|\hat{x}_{0}\right\|_{s}\right)+\sigma\left(\left\|\mathbf{w}_{[0, k-d-1]}\right\|_{s}\right) \\
& +\frac{L_{f}^{d}-1}{L_{f}-1}\left\|\mathbf{w}_{[k-d, k-1]}\right\|_{s}
\end{aligned}
$$

Now, define the new $\mathcal{K} \mathcal{L}$ function $\hat{\beta}(t, s)=\beta(t, 2 s)$ and $\theta(s)=\hat{\beta}(0, s)$. Based on this, the following property holds 
for any $t, s_{1}, s_{2} \in \mathbb{R}_{+}$.

$$
\begin{aligned}
\beta\left(t, s_{1}+s_{2}\right) & \leq \beta\left(t, 2 \max \left(s_{1}, s_{2}\right)\right) \\
& =\max \left(\beta\left(t, 2 s_{1}\right), \beta\left(t, 2 s_{2}\right)\right) \\
& =\max \left(\hat{\beta}\left(t, s_{1}\right), \hat{\beta}\left(t, s_{2}\right)\right) \\
& \leq \hat{\beta}\left(t, s_{1}\right)+\hat{\beta}\left(t, s_{2}\right) \\
& \leq \hat{\beta}\left(t, s_{1}\right)+\hat{\beta}\left(0, s_{2}\right) \\
& =\hat{\beta}\left(t, s_{1}\right)+\theta\left(s_{2}\right)
\end{aligned}
$$

Considering that $\left\|\hat{x}_{0}\right\|_{s} \leq\left\|x_{d}\right\|_{s}+\left\|e_{d}\right\|_{s}$ and based on the previous property for $s_{1}=\left\|x_{d}\right\|_{s}$ and $s_{2}=\left\|e_{d}\right\|_{s}$, we derive that

$$
\beta\left(k-d,\left\|\hat{x}_{0}\right\|_{s}\right) \leq \hat{\beta}\left(k-d,\left\|x_{d}\right\|_{s}\right)+\theta\left(\left\|e_{d}\right\|_{s}\right)
$$

From theorem 2, the following property can be proved

$$
\left\|e_{d}\right\|_{s} \leq \frac{L_{f}^{d}-1}{L_{f}-1}\left\|\mathbf{w}_{[0, d-1]}\right\|_{s}
$$

Taking into account equations (19)-(21), we have that

$$
\begin{aligned}
\left\|x_{k}\right\|_{s} \leq & \hat{\beta}\left(k-d,\left\|x_{d}\right\|_{s}\right)+\theta\left(\frac{L_{f}^{d}-1}{L_{f}-1}\left\|\mathbf{w}_{[0, d-1]}\right\|_{s}\right) \\
& +\sigma\left(L_{f}^{d}\left\|\mathbf{w}_{[0, k-d-1]}\right\|_{s}\right) \\
& +\frac{L_{f}^{d}-1}{L_{f}-1}\left\|\mathbf{w}_{[k-d, k-1]}\right\|_{s}
\end{aligned}
$$

Since $\left\|\mathbf{w}_{[0, d-1]}\right\|_{s},\left\|\mathbf{w}_{[0, k-d-1]}\right\|_{s}$ and $\left\|\mathbf{w}_{[k-d, k-1]}\right\|_{s}$ are lower that or equal to $\left\|\mathbf{w}_{[0, k-1]}\right\|_{s}$, then there exists a $\mathcal{K}$ function $\tilde{\sigma}$ such that

$$
\left\|x_{k}\right\|_{s} \leq \beta\left(k-d,\left\|x_{d}\right\|_{s}\right)+\hat{\sigma}\left(\left\|\mathbf{w}_{[0, k-1]}\right\|_{s}\right)
$$

Once the it is proved that the controlled system is ISS, in virtue of theorem 2 we have that if $\hat{x}_{j} \in X \ominus E$ for all $j \geq 0$, then since $e_{k} \in E$,

$$
x_{k}=\hat{x}_{k-d}+e_{k} \in X
$$

for all $k \geq d$.

The main consequence of this result is that we can design a control law for a modified uncertain system without delay and this control law together with the dead-time compensator will robustly stabilize the uncertain dead-time system.

Remark. 1: Due to the global nature of the Lipschitz constant, the bounds derived from this might be quite conservative. Then a suitable choice of the norms and an adhoc parameterization of the plant may lead to reduce the conservativeness.

Remark. 2: A practical procedure to design the proposed control structure based on the dead-time compensator may be the following: first a control law is calculated for the system without delay in such a way that the size of the admissible uncertainty $\hat{\gamma}$ is maximized . Then the Lipschitz constant of the controlled system is calculated. Notice that this is possible since the controller is already calculated, and this constant is potentially quite smaller that the constant calculated for the open-loop system. Finally, given the estimated bound of the uncertainty $\gamma$, the maximum allowable delay is calculated as $d_{\max }=\frac{\hat{\gamma}}{L_{f}^{d}}$. If $d$ is lower than or equal to $d_{\max }$, then the plant can be robustly stabilized by our control structure.

\section{A CASE Study}

This section presents a simulation case study that illustrates the proposed approach. The system under study is a continuously stirred tank reactor (CSTR), that consists of a tank in which a irreversible exothermic decomposition reaction $A \rightarrow B$ takes place [15].

The temperature $T$ inside the reactor is controlled using a cold fluid which circulates in the reactor jacket. The refrigerant flow is constant and its temperature $T_{c}$ can be controlled in an coolant system located at a certain distance from the reactor. As this system has a fast time constant if compared to the reactor its model is a simple gain, therefore $T_{c}$ can be modelled as:

$$
T_{c}(t)=r(t-L)
$$

where $r(t)$ is the reference of the coolant system and $L$ is the delay caused by mass transportation.

The following equations describe the dynamics of the process (reactor plus coolant system) considering $T(t)$ as process output and $r(t)$ as manipulated variable, and assuming: (i) a perfect mixture inside the tank, (ii) a first order reaction kinetics, (iii) the tank volume $V$ (and its level) is constant, and (iv) a negligible dissipation to the external environment.

$$
\begin{aligned}
\frac{d C_{A}(t)}{d t}= & \frac{q}{V}\left(C_{A f}-C_{A}(t)\right)-k_{0} e^{\frac{-E}{R T(t)}} C_{A}(t) \\
\frac{d T(t)}{d t}= & \frac{q}{V}\left(T_{f}-T(t)\right)+\frac{-\Delta H_{r}}{\rho C_{p}} k_{0} e^{\frac{-E}{R T(t)}} C_{A}(t) \\
& +\frac{U A}{V \rho C_{p}}(r(t-L)-T(t))
\end{aligned}
$$

where $C_{A}$ is the concentration of product $\mathrm{A}$ inside the tank, $C_{A f}, T_{f}$ and $q$ are respectively the concentration, temperature and flow of the influx of A. Moreover, $\rho=1000 \mathrm{~g} / \mathrm{l}$, $k_{0}=7.210^{10} 1 / \mathrm{min}, U A=510^{4} \mathrm{~J} / \mathrm{minK}, E / R=8750 \mathrm{~K}$, $-\Delta H_{r}=510^{4} \mathrm{~J} / \mathrm{mol}, C_{p}=0.239 \mathrm{~J} / \mathrm{gK}$.

The operation point of the reactor is obtained with the inputs $q=100 \mathrm{l} / \mathrm{min}, T_{f}=350 \mathrm{~K}, V=100 \mathrm{l}$ and $C_{A f}=$ $1.0 \mathrm{~mol} / \mathrm{l}$, achieving $C_{A}^{o}=0.5 \mathrm{~mol} / \mathrm{l}, T^{o}=350 \mathrm{~K}$ and $r^{\circ}=$ $300 K$ [16]. Note that this equilibrium point is unstable.

A normalized model can be obtained using the following change of variables:

$$
x(1)=\frac{C_{A}-0.5}{0.5} ; x(2)=\frac{T-350}{20} ; u=\frac{r-300}{20}
$$



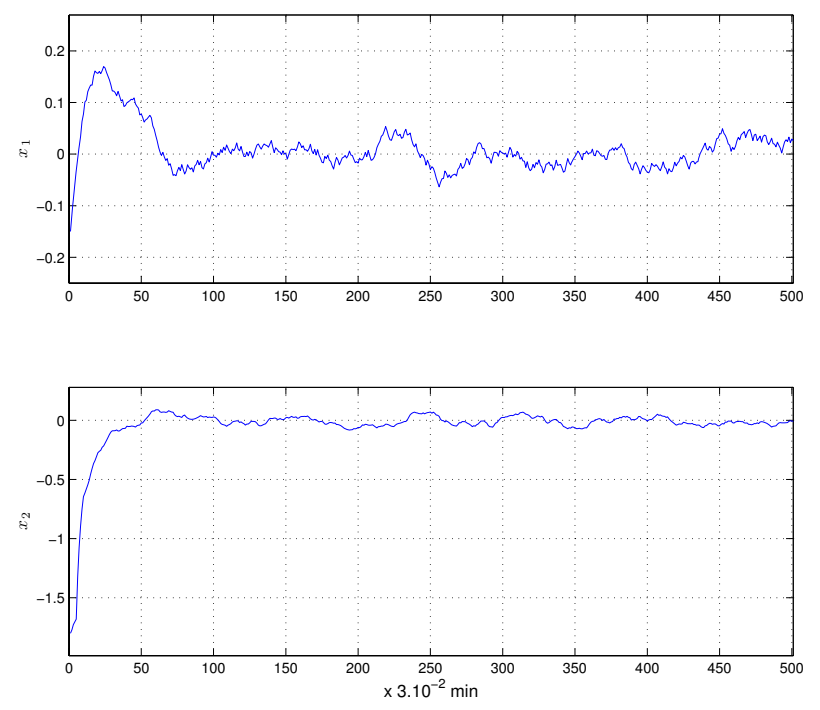

Fig. 2. Closed loop performance with delay compensation.

where $u$ is the control action and $x(1), x(2)$ are the states of the plant, which are limited in

$$
x(1) \in[-0.21 ; 0.21], x(2) \in[-2 ; 0.25] .
$$

Discretizing the model with a sampling time $T_{s}=0.03$ min and considering the uncertainties $w_{k}$, the discrete model is then giving by:

$$
x_{k+1}=f\left(x_{k}, u_{k-d}\right)+w_{k}
$$

where $d=5$ is the delay in samples and $x_{k}=\left[x(1)_{k} x(2)_{k}\right]^{\prime}$.

For a null dead-time $(d=0)$ this process was controlled in [16] using a discrete state feedback law $u_{k}=K x_{k}$, where $K=[-1.5488-3.4658]$, in such a way that the closed-loop system has robust stability for $\left\|\hat{w}_{k}\right\| \leq 0.1218$.

Thus, applying the results in section IV, $L_{f}=1.6$, and

$$
\left\|w_{k}\right\|=\frac{\left\|\hat{w}_{k}\right\|}{(1.6)^{5}} \leq 0.0116
$$

which means that the dead-time system is robust stable for this uncertainty if a dead-time compensator is used together with a controller $u=K \hat{x}$. To illustrate the performance of this controller, a simulation is shown in figure 2 for $x_{0}=$ $(-0.15,1.8)$ and the signal $w_{k}$ generated randomly in the defined range.

As can be seen the closed-loop system is stable and because of the conservationism of the Lipschitz constant computation, the dynamic behavior has no oscillations.

\section{CONCLUSIONS}

This paper has presented a predictor based approach to design robust controllers for nonlinear systems. The predictor scheme allows to compute an equivalent uncertainty dead-time free system and to bound the equivalent model uncertainty. This equivalent dead-time system is then used to compute a stabilizing control law which guaranty robust stability for the original one. As a dead-time free system is used in this last step the control design is simplified. A simulation case study was used to illustrate the theoretical results.

\section{REFERENCES}

[1] J. E. Normey-Rico and E. F. Camacho, Control of dead-time processes. Springer Verlag, 2007.

[2] Z. J. Palmor, Time-delay compensation \& Smith predictor and its modifications. CRC Press and IEEE Press, 1996, ch. The control handbook, chap. 10.8, pp. 224-237.

[3] O. J. M. Smith, "Closed control of loops with dead-time," Chemical Engeneering Progress, vol. 53, no. 5, pp. 217-219, 1957.

[4] Q.-C. Zhong, Robust Control of Time-delay Systems. Springer-Verlag London, 2006.

[5] L. Zaccarian, D. Nesic, and A. R. Teel, "L2 anti-windup for linear dead-time systems," Systems \& Control Letters, vol. 52, no. 12, pp. 1205-1217, 2005.

[6] K. Yakoubi and Y. Chitour, "On the stabilization of linear discrete-time delay systems subject to input saturation," in Advanced Strategies in Control Systems with Input and Output Constraints, ser. Lecture Notes in Control and Information Sciences, S. Tarbouriech, G. Garcia, and A. Glattfelder, Eds. Springer Berlin, 2007, vol. 346, pp. 421-455.

[7] J. M. G. da Silva, I. Ghiggi, and S. Tarbouriech, "Non-rational dynamic output feedback for time delay systems with saturating inputs," International Journal of Control, vol. 81, no. 4, pp. 557-570, 2008.

[8] B. Zhou, G. R. Duan, and Z. Lin, "Global stabilization of the double integrator system with saturation and delay in the input," IEEE Transactions on Circuits and Systems I: Regular Papers, vol. 57, no. 6, pp. 1371-1383, 2010.

[9] T. L. M. Santos, D. Limon, T. Alamo, and J. E. Normey-Rico, "Robust tube based model predictive control for constrained systems with deadtime," in UKACC International Conference on Control, 2010.

[10] D. Limón, T. Álamo, and E. Camacho, "Input-to-state stable MPC for constrained discrete-time nonlinear systems with bounded additive uncertainties," in Proceedings of the 41st IEEE, Conference on Decision and Control, Las Vegas, Nevada USA, December 2002, pp. 4619-4624.

[11] C. V. Rao, S. J. Wright, and J. B. Rawlings, "Application of interiorpoint methods to model predictive control," Journal of optimization theory and applications, vol. 99, no. 3, pp. 723-757, 1998.

[12] H. K. Khalil, Nonlinear systems. Prentice Hall, 1996.

[13] Z.-P. Jiang and Y. Wang, "Input-to-state stability for discrete time non linear systems," Automatica, no. 37, pp. 857-869, 2001.

[14] D. Limon, T. Alamo, D. M. Raimondo, D. M. de la Peña, J. M. Bravo, A. Ferramosca, and E. F. Camacho, "Input-to-state stability: A unifying framework for robust model predictive control," Lecture Notes in Control and Information Sciences, vol. 20, no. 3, pp. 1-26, 2010.

[15] D. E. Seborg, T. Edgar, and D. Mellichamp, Process Dynamics and Control. Wiley, 1989.

[16] D. Limón, "Control predictivo de sistemas no lineales con restricciones: estabilidad y robustez," Ph.D. dissertation, Escuela Superior de Ingenieros - Universidad de Sevilla, 2002. 